\title{
Electromagnetically induced transparency-based gas detector design using Michelson interferometer
}

\author{
Karim Abbasian ${ }^{1 *}$ and Mohammad Hossein Abdollahi ${ }^{2}$
}

\begin{abstract}
In this paper, we have designed an all-optical controllable gas detector by doping 3-level $\wedge$ type nanocrystals in the moving arms' mirror of Michelson interferometer and used electromagnetically induced transparency (EIT) phenomenon to change its refractive index. By this means, we have created a controllable phase difference between light beams in two arms of the Michelson interferometer, where reflection phase of the EIT-based mirror changes about $\pi$ radiant. Also, the signal reflection from EIT-based mirror changes between $0 \%$ and 100\% approximately, while the second arm's signal is reflected completely. This EIT-based mirror's refractive index change can be a good alternative for conventional Michelson interferometer-based gas detector with one mechanical moving arm mirror (Undergraduate Instrumental Analysis, 6th edn. Marcel Dekker, New York, 2005), where long response time and unfix moving speed were its main drawbacks. While, in this scheme, not only these disadvantages are removed but also the response time can reach the electron transient time between the atomic energy levels. Then, by this all-optical tunable gas detector, we have achieved many modifications such as response time in sub-nanoseconds, high resolution, and high accuracy, or less cross sensitivity to other gas species.
\end{abstract}

Keywords: Hydrogen sulfide $\left(\mathrm{H}_{2} \mathrm{~S}\right)$ sensor, Gas sensor, EIT, Michelson interferometer

\section{Background}

The hydrogen sulfide $\left(\mathrm{H}_{2} \mathrm{~S}\right)$ is a colorless, toxic, and flammable gas, which is partially responsible for the foul odor of rotten eggs. It often results from the bacterial break down of sulfates in organic matter in the absence of oxygen, such as in swamps and sewers. It also occurs in volcanic gases, natural gas, and some well waters $[1,2]$.

Small amounts of hydrogen sulfide occur in crude petroleum, but natural gas can contain up to $90 \%$. Also, the $\mathrm{H}_{2} \mathrm{~S}$ is emitted from volcanoes and hot springs, and generated as common by-product of many industrial processes including oil and gas exploration and production operations, where, it probably arises via the hydrolysis of sulfide minerals, i.e., $\mathrm{MS}+\mathrm{H}_{2} \mathrm{O} \rightarrow \mathrm{MO}+\mathrm{H}_{2} \mathrm{~S}$. Normal concentration of the $\mathrm{H}_{2} \mathrm{~S}$ in clean air is about 0.0001 to

\footnotetext{
* Correspondence: k_abbasian@tabrizu.ac.ir

${ }^{1}$ School of Engineering Emerging-Technologies, University of Tabriz, Tabriz 51666, Iran

Full list of author information is available at the end of the article
}

$0.0002 \mathrm{ppm}$. Owing to its high toxicity, concentration level above $10 \mathrm{ppms}$ is considered dangerous, and levels above $100 \mathrm{ppm}$ are potentially fatal.

Early detection of the $\mathrm{H}_{2} \mathrm{~S}$ at concentrations of 10 $\mathrm{ppm}$ in air is essential to prevent its toxic influence at higher concentrations. For this reason, many reports on $\mathrm{ZnO}$ sensors [1], resistance sensor by $\mathrm{n}-\mathrm{SnO}_{2} /$ $\mathrm{p}-\mathrm{CuO}$ heterojunctions (p-type $\mathrm{CuO}$ grains around n-type $\mathrm{SnO}_{2}$ grains) [3], detector design using a nanocrystalline $\mathrm{WO}_{3}$ [4], and many other works on $\mathrm{H}_{2} \mathrm{~S}$ gas-sensing materials as $\mathrm{SnO}_{2}-\mathrm{CuO}$ [3-8], CuO$\mathrm{SnO}_{2}-\mathrm{ZnO}$ [9], $\mathrm{SnO}_{2}-\mathrm{Pd}$ [10], modified $\mathrm{BaTiO}_{3}$ [11], $\mathrm{SnO}_{2}-\mathrm{Al}_{2} \mathrm{O}_{3}$ [12], $\mathrm{SnO}_{2}-\mathrm{CuO}-\mathrm{SnO}_{2}$ [13], and $\mathrm{ZnSb}_{2} \mathrm{O}_{6}$ [14] have been done for the detection of $\mathrm{H}_{2} \mathrm{~S}$. However, these non-optical point detectors which are electrochemical and semiconductor devices have limited success, although used in a number of different strategies, because they have limitations such as being non-intrusive, poor selectivity and sensitivity, and limited response time compare to the semiconductorbased gas sensors. Also, these sensors have other 
problems; for instance, the gas of interest should reach the detector's surface and chemically react with it. Also, sensor drift, noise effects, static system of gas sensing, operation at different temperatures, cross sensitivity to other species, and a tendency of poisoning are other drawbacks.

In another work, long open-path $\mathrm{H}_{2} \mathrm{~S}$ sensor based on tunable diode laser absorption spectroscopy was proposed as an all optical gas detector in open air [15]. This optical gas sensor was not only expensive and complicated but also has a low signal-to-noise ratio (SNR) which is a serious problem at low gas concentrations.

On the other hand, electromagnetically induced transparency (EIT) is one of the interesting phenomena of the light-matter interaction which modifies the material property by applying an optical control field. In other words, it changes the absorption and refractive coefficients of the material to the probe field. Then, we can change the absorption and refractive indexes of the material at the resonant frequency.

So, we have introduced an all-optical tunable gas detector with the Michelson interferometer [16] which modifies the SNR ratio and does not have any of the above-mentioned disadvantages.

\section{Methods}

As we know, infrared absorption can be used to provide quantitative measurements of different compounds. A beam of light absorption in an absorbing medium results in its attenuation.

According to the Beer-Lambert law, the relationship between the absorbance $(A)$ and density $(N)$ of an absorbing species of molecules is linear.

$$
A=\sigma N L=\alpha L
$$

where $A, \sigma\left(\mathrm{cm}^{2}\right), L(\mathrm{~cm}), N\left(\mathrm{~cm}^{-3}\right)$, and $\alpha\left(\mathrm{cm}^{-1}\right)$ are the measured absorbance, the frequency-dependent absorption cross-section, path length, analytic particle density, and the attenuation coefficient, respectively, as illustrated in the absorption cell of Figure 1. Transmittance, $T$, is defined by

$$
T=I_{\mathrm{L}} /_{I_{\mathrm{o}}},
$$

where $I_{\mathrm{L}}$ and $I_{0}$ are the attenuated and initial intensities in the sample, and the relation between $A$ and $T$ is

$$
A=-\operatorname{Ln} T=-\operatorname{Ln}\left(I_{\mathrm{L}} / I_{\mathrm{o}}\right)
$$

By measuring the absorbed light intensity and applying the Beer's law, we can determine the concentration of the analyze atom or molecule. A simple principle of gas detection, based on absorption, has been demonstrated in Figure 1. This setup has been afforded by the advent of tunable laser sources, in particular, tunable semiconductor diode lasers. The combination of high intensity and narrow line width makes lasers ideal light sources for such applications [2].

In the case of weak absorption, the sensitivity of this setup might be insufficiently accurate to obtain quantitative results, which known as its disadvantage. Using the Michelson interferometer is a solution to improve SNR [1]. This system consists of four optical arms, with a beam splitter at their intersection point. Radiation passes down the first arm and is separated by beam splitter into two perpendicular beams of equal intensity which passes down into vertical and horizontal arms of the spectrometer. At the ends of these arms, the two beams are reflected by mirrors back to the beam splitter, where they recombine and are reflected together onto the detector. One of the mirrors is fixed in its position, while the other mirror moves from the central position or zero path difference (ZPD), changing the path length of that arm.

In practice, one of the mirrors in one arm is kept stationary, and the second mirror in the other arm is moved slowly. As the moving mirror moves, the net signal falling on the detector is a cosine wave with the usual maxima and minima when plotted against the

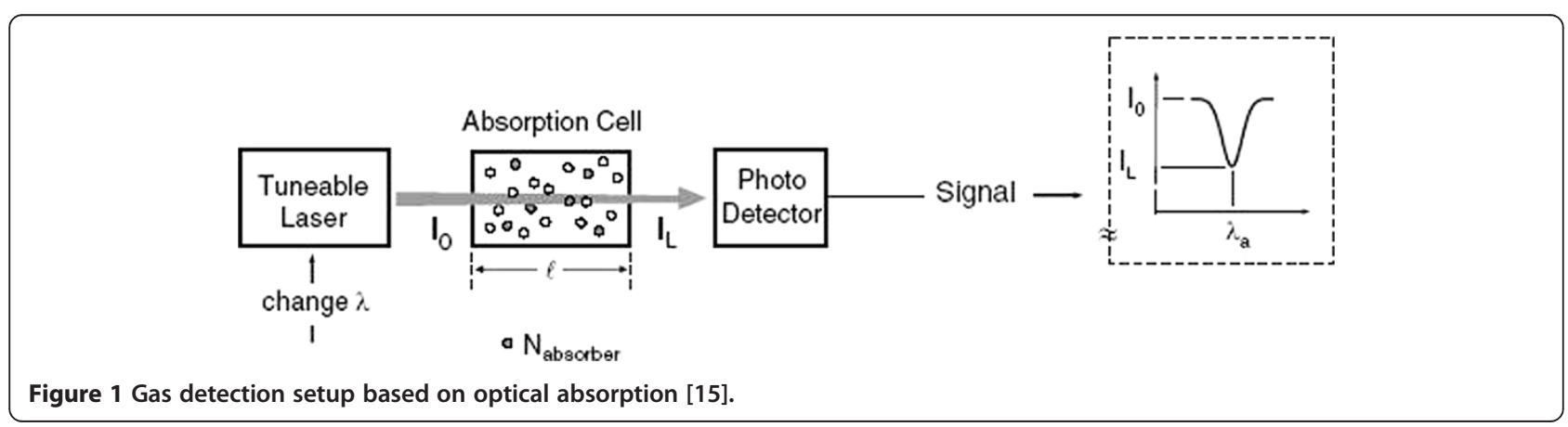




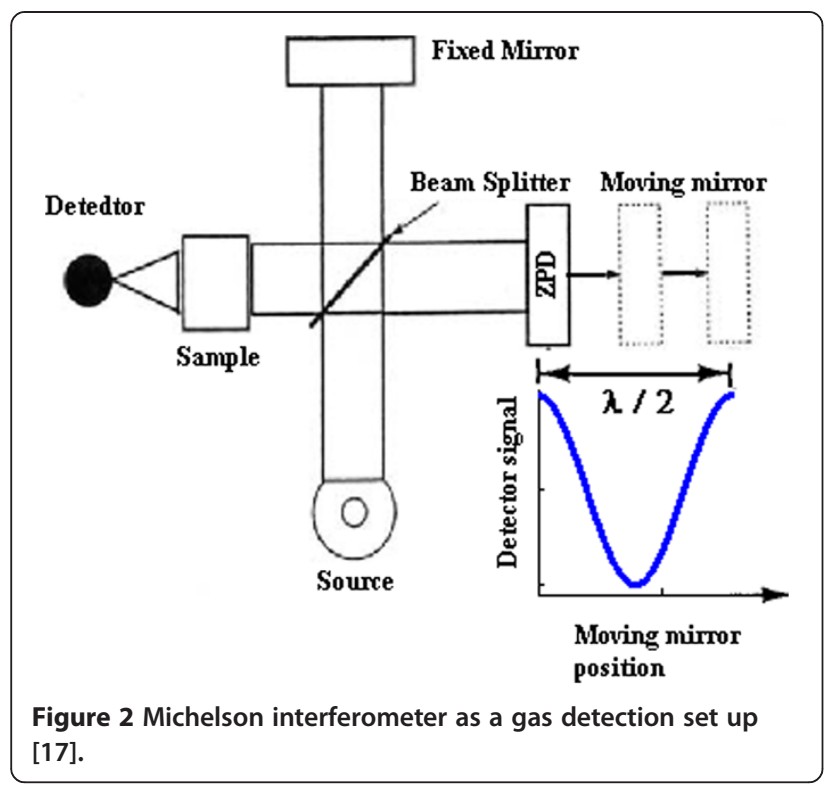

travel of the mirror as shown in Figure 2. The intensity of the reached wave to the detector is given by

$$
I=2 I_{\mathrm{o}}(1+\cos (4 \pi d / \lambda))
$$

where $d$ and $I_{0}$ are path difference from ZPD position of the moving mirror and the light intensity of the arms at the beam splitter [17].

The disadvantage of this setup is the mechanical problem when moving the mirror at a controlled, known steady, velocity; position variations due to temperature changes, vibrations, and other environmental effects must be corrected. On the other hand, many faults might occur in the moving parts due to mechanical motion.

In this paper, to resolve the disadvantage mentioned in the last part of the previous paragraph, we have replaced the moving mirror with an all-optical tunable slab mirror by EIT phenomenon with doped 3-level $\Lambda$-type nanocrystals. By this means, we have achieved a required phase difference by varying the refraction index instead of the mechanical path difference created between arms.

Then, we have developed a mathematical method to describe the effect of the parameters of 3-level particles added in the considered slab on the optical characteristics of the designed device (mirror). Figure 3 shows the proposed all-optical tunable mirror with 3-level nanoparticles including the probe and control fields and decay rates. In this figure, the control and probe fields have been applied to $|\mathrm{c}\rangle-|\mathrm{a}\rangle$ and $|\mathrm{b}\rangle-|\mathrm{a}\rangle$ atomic transitions, respectively. Due to the control field application, the optical characteristic of the mirror is changed for the probe field. In the following, the theoretical calculation to describe the proposed system is presented.

After some mathematical manipulation, which is well known in the quantum optics, the following matrix form the Hamiltonian, and the density matrix are given as follows.

$$
H=\hbar\left[\begin{array}{ccc}
\omega_{b} & 0 & -\frac{\wp_{a b} \varepsilon}{2 \hbar} e^{i v_{p} t} \\
0 & \omega_{c} & -\frac{1}{2} \Omega_{c} e^{i \phi_{c}} e^{i v_{c} t} \\
-\frac{\wp_{a b} \varepsilon}{2 \hbar} e^{-i v_{p} t} & -\frac{1}{2} \Omega_{c} e^{i \phi_{c}} e^{-i v_{c} t} & \omega_{a}
\end{array}\right]
$$

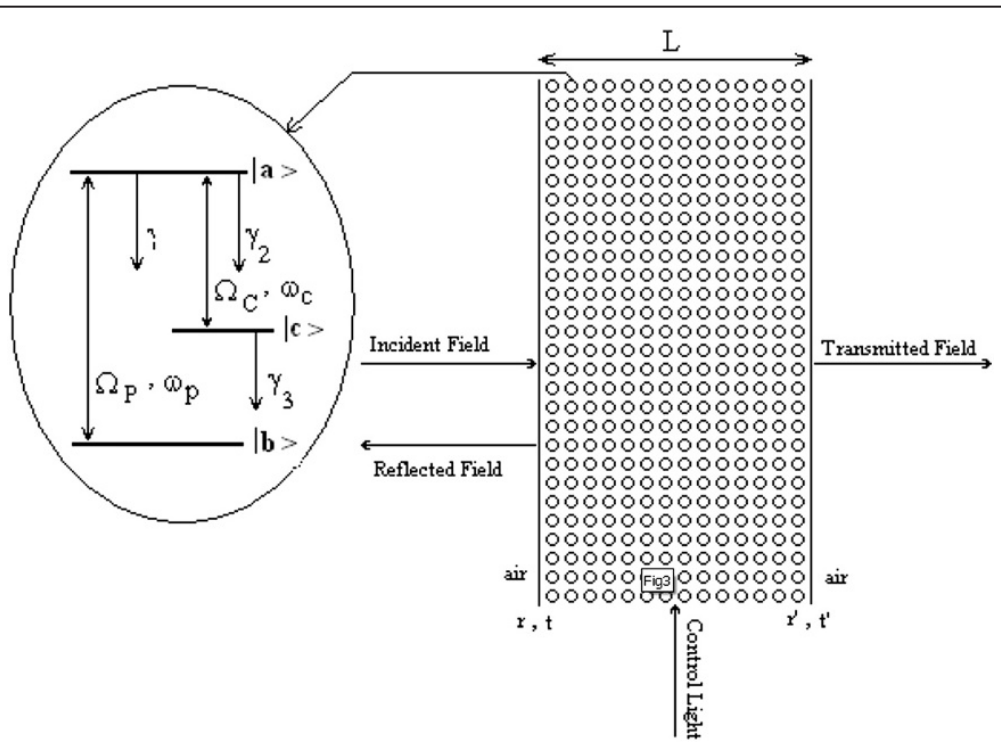

Figure 3 Schematic of the proposed all-optical tunable mirror with 3-level $\Lambda$-type quantum dot dopants [18]. 


$$
\rho=\left[\begin{array}{ccc}
\rho_{b b} & \tilde{\rho}_{c b}^{*} e^{i \omega_{c b} t} & \tilde{\rho}_{a b}^{*} e^{i \omega_{a b} t} \\
\tilde{\rho}_{c b} e^{-i \omega_{c b} t} & \rho_{c c} & \tilde{\rho}_{a c}^{*} e^{i \omega_{a c} t} \\
\tilde{\rho}_{a b} e^{-i \omega_{a b} t} & \tilde{\rho}_{a c} e^{-i \omega_{a c} t} & \rho_{a a}
\end{array}\right],
$$

where $\omega_{\mathrm{a}}, \omega_{\mathrm{b}}, \omega_{\mathrm{c}}, \gamma_{\mathrm{ab}}, \varepsilon, v_{\mathrm{p}}, v_{\mathcal{O}} \varphi_{\mathrm{c}}$, and $\Omega_{\mathrm{c}}$ are corresponding frequencies to three levels ( $\mathrm{a}, \mathrm{b}$, and c), coherency decay rate of the transition $|a\rangle-|b\rangle$, amplitude of the probe field, probe field frequency, control field frequency, control field phase, and Rabi frequency of the control field, respectively. $\omega_{\mathrm{cb}}, \omega_{\mathrm{ab}}$, and $\omega_{\mathrm{ac}}$ are the corresponding frequencies of the transitions between the levels in the presented model. Based on the time development equation of the density matrix [19] with differential motion equations and assuming appropriate boundary conditions, the below matrix equation with the following matrices can be obtained:

$$
\dot{R}=-M R+A,
$$

with

$$
\begin{aligned}
& R=\left[\begin{array}{c}
\tilde{\rho}_{\mathrm{ab}} \\
\tilde{\rho}_{\mathrm{cb}}
\end{array}\right] \\
& M=\left[\begin{array}{cc}
i \Delta+\gamma_{1} & -\frac{i}{2} \Omega_{\mathrm{c}} e^{-i \phi_{\mathrm{c}}} \\
-\frac{i}{2} \Omega_{\mathrm{c}} e^{i \phi_{\mathrm{c}}} & i \Delta+\gamma_{2}
\end{array}\right] \\
& A=\left[\begin{array}{c}
i \wp_{\mathrm{ab}} \varepsilon / 2 \hbar \\
0
\end{array}\right],
\end{aligned}
$$

where $\gamma_{1}$ and $\gamma_{2}$ are the decay rates of the $\rho_{\mathrm{ab}}$ and $\rho_{\mathrm{cb}}$ density matrix elements. Also, $\Omega_{\mathrm{c}}, \wp_{\mathrm{ab}}$ and $\Delta$ are the Rabi frequency of the control field, dipole moment element of the $|a>-| b>$ transition, and detuning of the probe field from resonance frequency, respectively.

Then, the optical susceptibility can be obtained with manipulating of the relationship between polarization and the dipole moment element as follows:

$$
X=\frac{P}{\varepsilon_{0} E}=\frac{2 N_{\mathrm{a}} \wp^{2} \rho_{\mathrm{ab}}}{\varepsilon_{0} \Omega_{\mathrm{p}} \hbar},
$$

where $\Omega_{\mathrm{p}}$ and $N_{\mathrm{a}}$ are the Rabi frequency of the probe field and the density of 3-level atoms, respectively. According to basic concepts, the optical loss or gain and induced refraction index, using the real and imaginary parts of the susceptibility, can be found as follows:

$$
\begin{aligned}
& \alpha=\frac{\kappa}{2} \chi^{\prime \prime} \\
& \delta n=n \frac{X^{\prime}}{2},
\end{aligned}
$$

where $\mathrm{X}^{\prime}, \mathrm{X}^{\prime \prime}, \kappa$, and $n$ are the real and imaginary parts of the optical susceptibility, wave number, and refractive index, respectively $[18,19]$. According to the basic concepts in classical optics and basic relations in homogeneous, linear, and isotropic media, the following relations are presented for the reflected part of the incident light. The phase difference of the light wave
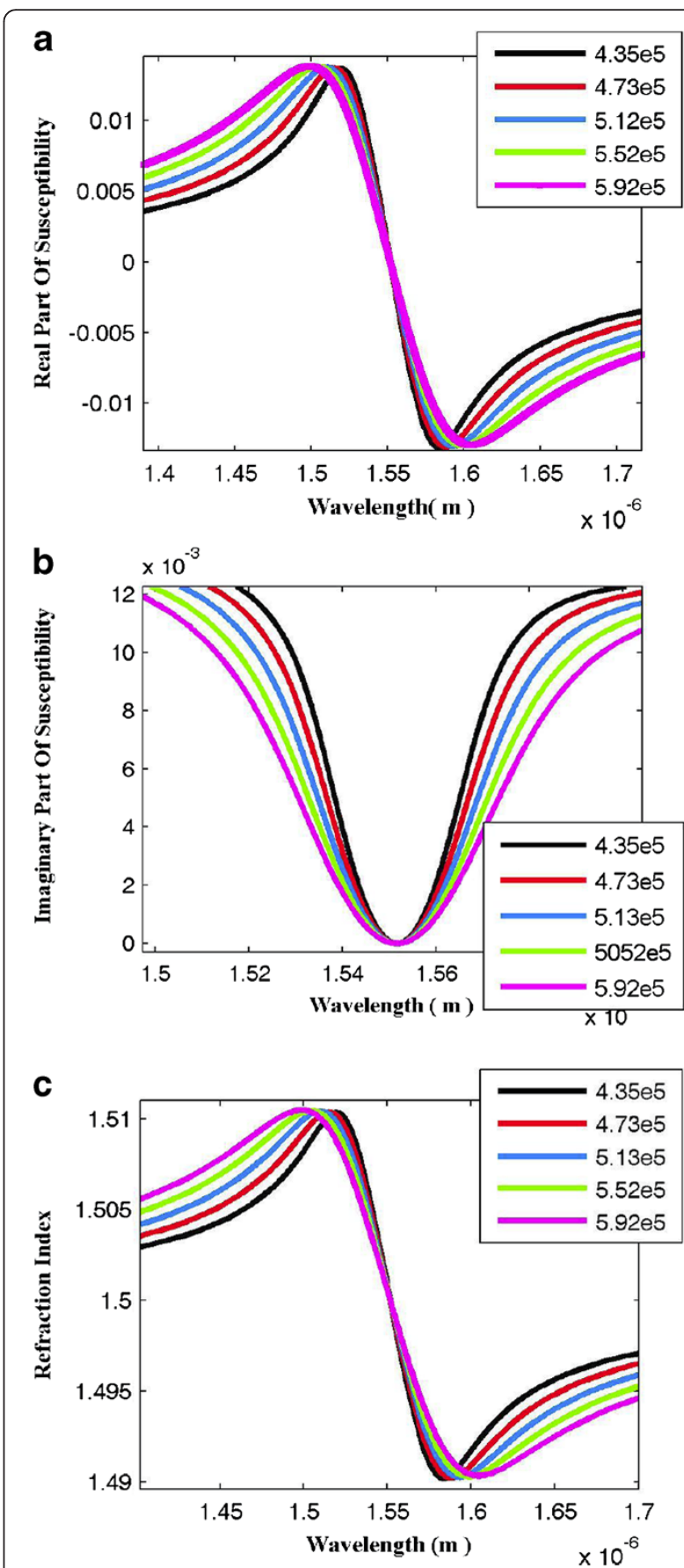

Figure 4 The optical susceptibility vs. wavelength for different control field amplitudes as a parameter. (a) The real part, (b) imaginary part, and (c) refraction index. $E_{\mathrm{p}}=1 \mathrm{~V} / \mathrm{m}, \mathrm{N}_{\mathrm{a}}=$ $7.5 e 16 \mathrm{~cm}^{-3}, \gamma_{1}=5 e 11 \mathrm{~s}^{-1}, \gamma_{2}=\gamma_{3}=1 e 7 \mathrm{~s}^{-1}, \wp_{\mathrm{ab}}=5 e^{-} 9 e^{-} \mathrm{cm}$. 
propagating through the media and conservation of the reflection and transmission coefficients are as follows [18].

$$
\begin{aligned}
& \delta=2 \pi \frac{n_{r} L}{\lambda} \\
& \left\{\begin{array}{c}
r^{\prime}=-r \\
r^{2}+t t^{\prime}+\alpha=1
\end{array}\right.
\end{aligned}
$$

The following relation can be derived for the reflected field amplitude.

$$
E_{\mathrm{R}}=E_{\mathrm{I}} \frac{r\left(1-e^{i 2 \delta}\right)}{1-\mathrm{Re}^{i 2 \delta}}
$$

where $R=|r|^{2}$ is the intensity reflection coefficient at individual interfaces. $E_{\mathrm{I}}$ and $\delta$ are the incident electric field and the gained phase delay in propagation of the slab length, respectively. Then, the absolute value of

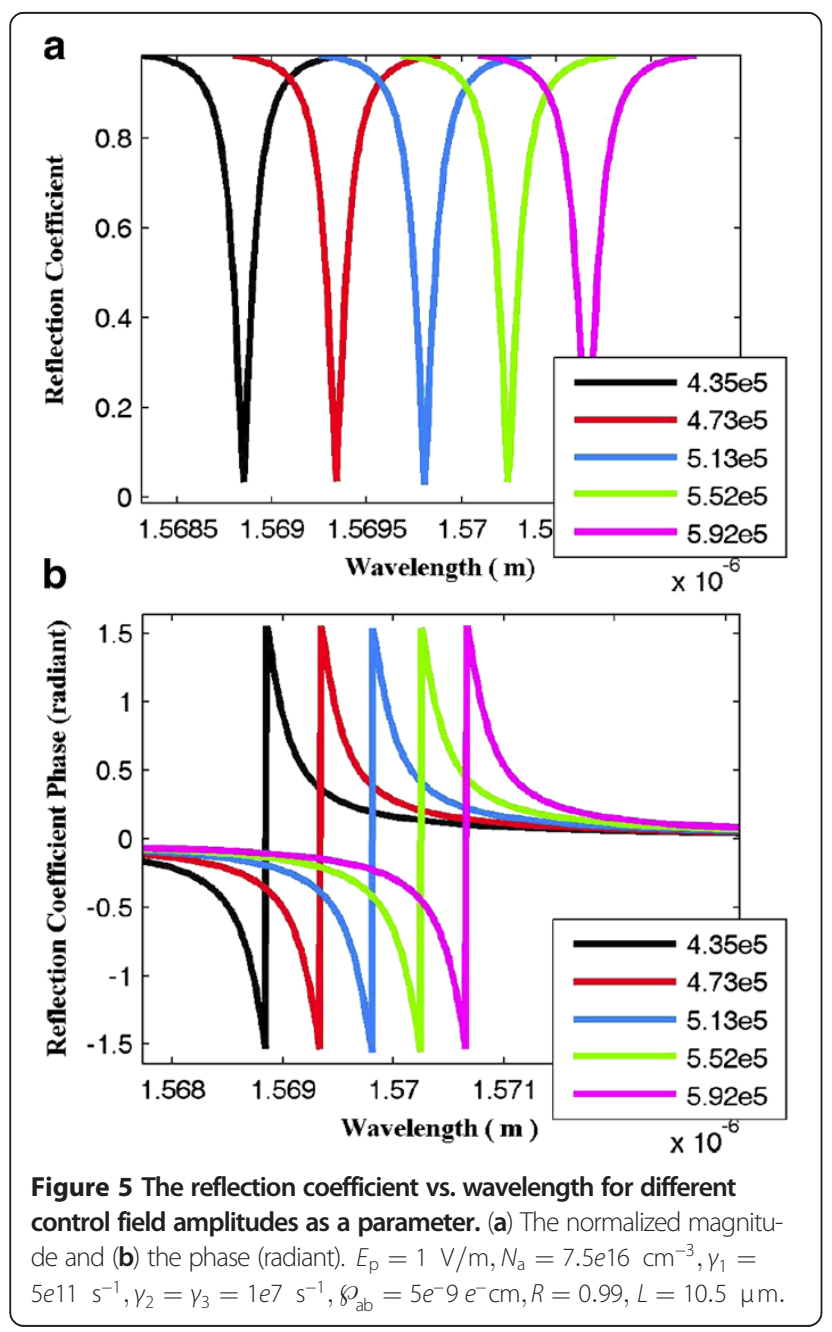

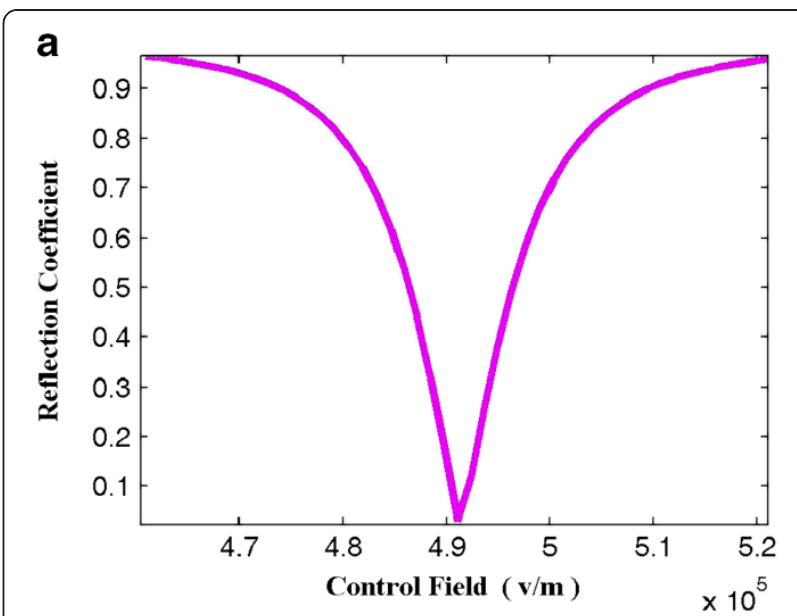

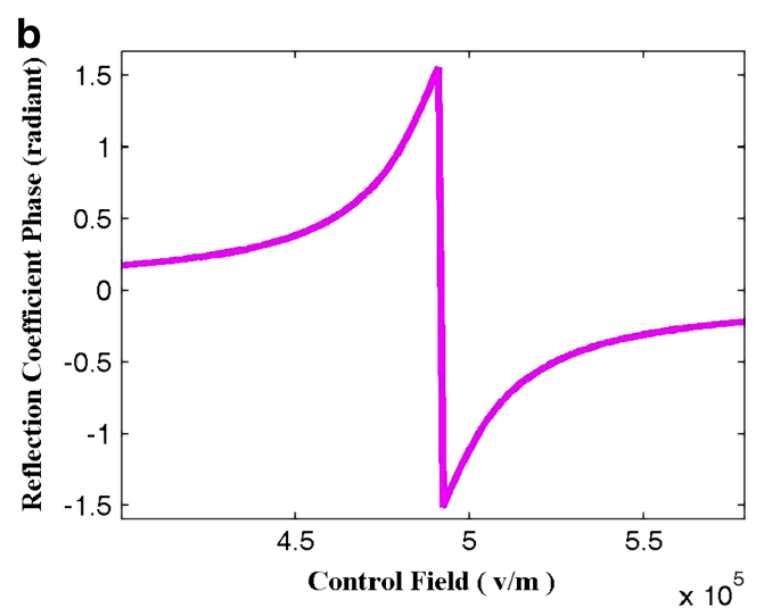

Figure 6 The reflection coefficient variation vs. the control field amplitude at wavelength of $1.57 \mu \mathrm{m}$. (a) The normalized magnitude. (b) The phase (radiant). $E_{\mathrm{p}}=1 \mathrm{~V} / \mathrm{m}, N_{\mathrm{a}}=7.5 e 16 \mathrm{~cm}^{-3}$, $\gamma_{1}=5 e 11 \mathrm{~s}^{-1}, \gamma_{2}=\gamma_{3}=1 e 7 \mathrm{~s}^{-1}, \wp_{a b}=5 e^{-} 9 e^{-} \mathrm{cm}, R=0.99$, $L=10.5 \mu \mathrm{m}$.

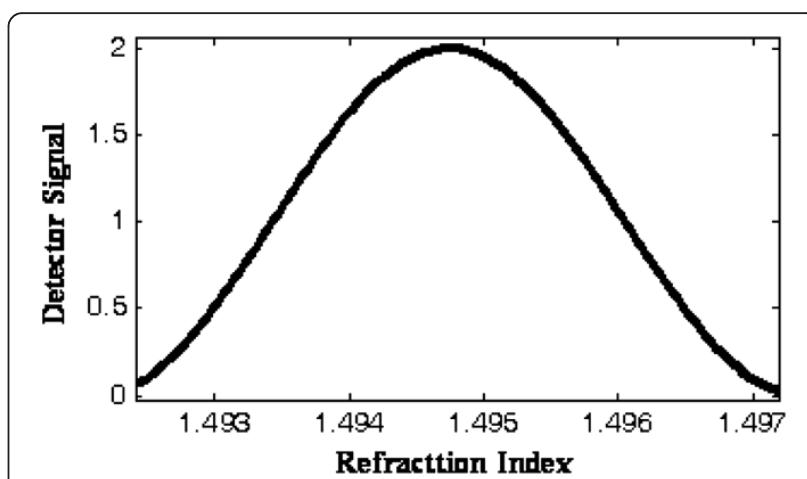

Figure 7 Normalized detected signal of the proposed structure vs. refractive index variation of the EIT-based mirror. The wavelength is $1.57 \mu \mathrm{m} . E_{\mathrm{p}}=1 \mathrm{~V} / \mathrm{m}, \mathrm{N}_{\mathrm{a}}=7.5 \mathrm{e} 16 \mathrm{~cm}^{-3}, \gamma_{1}=5 \mathrm{e} 11$ $\mathrm{s}^{-1}, \gamma_{2}=\gamma_{3}=1 e 7 \mathrm{~s}^{-1}, \wp_{\mathrm{ab}}=5 e^{-} 9 e^{-} \mathrm{cm}, R=0.99, L=10.5 \mu \mathrm{m}$. 
the reflection coefficient and its phase can be extracted as follows [18]:

$$
\left|r_{E}\right|=\left|\frac{E_{\mathrm{R}}}{E_{\mathrm{I}}}\right|=\frac{\sqrt{\left[((1+R)(1-\cos 2 \delta))^{2}+((R-1) \sin 2 \delta)^{2}\right]}}{(1-R \cos 2 \delta)^{2}+R^{2} \sin ^{2} 2 \delta}
$$

$$
\phi_{\frac{E_{R}}{E_{1}}}=\tan ^{-1}\left[\left(\frac{R-1}{1+R}\right) \cot \delta\right]
$$

\section{Results and discussion}

In this section, we have considered the effect of the applied control field on optical susceptibility. The refractive index of the mirror is shown in Figure 4. Then, we have investigated effect of the applied control field on the magnitude and phase of the reflection coefficient, which is illustrated in Figure 5. It is well known that increasing the control field intensity broadens the EIT window; therefore, the slope of the refractive index decreases. We have shown that by changing the control field (illustrated in the figure legend), the magnitude and phase of the reflection coefficient changed at given wavelength as resonant wavelength of $\mathrm{H}_{2} \mathrm{~S}$ at 1.57 micron. So, by applying the control light, optically tunable capability of the proposed mirror is obtained and illustrated in the EIT window.

Figure 5 shows that by increasing the control field, the reflection profile is shifted to long wavelengths. This effect can be described in terms of upper level splitting. By increasing the control field, the upper energy level splits more; then, the probe field with smaller energy can be absorbed. Thus, the resonant wavelength shifts to long wavelengths.

Reflection coefficient variation at wavelength of 1.57 $\mu \mathrm{m}$ versus control field amplitude is shown in Figure 6. It is observed that the signal reflection from the EIT-based mirror changes between $0 \%$ and 100\% approximately, while the second arm's signal is reflected completely. Also, the reflection phase of the EIT-based mirror changes about $\pi$ radiant.

Figure 7 demonstrates the normalized detector signal versus refractive index changes of EIT-based mirror. It has been demonstrated that with this given structure and with the considered parameters, the detector signal changes between zero and twice the initial intensity. Also, we should mention that according to Figure 3, EIT window can be shifted and broadened; so, this structure not only can be used for detecting other gases with different absorption wavelength but also enhances performance problems such as unfixed speed of mechanical moving.

\section{Conclusion}

Valuable researches have been done on EIT, and its applications have been extended approximately to all fields of optics. Developing all optical tunable devices, such as sensors, is one of the fields which replace electronic sensors as an appropriate alternative.

In this article, we have used $\Lambda$ type 3-level quantum nanoparticles doped into a thin solid medium as mirror of Michelson interferometer to achieve EIT conditions. EIT phenomenon by doped 3-level quantum dots implements quantum interference to change the refraction index of the mirror and consequently the $\pi$ radiant phase difference of the light wave propagating through it. Also, the signal reflection from the EIT-based mirror has changed between $0 \%$ and $100 \%$ approximately. Thus, we can use refractive index changing instead of mechanical moving of the interferometer's mirror.

\section{Competing interest}

The authors declare that they have no competing interests.

\section{Authors' contributions}

MHA started the research with all-optical sensor design studies with consultations to KA. He also prepared the simulation results. KA taught him the simulation and contributed in simulating and in designing the proposed structure. KA also drafted the manuscript. All authors read and approved the final manuscript.

\section{Authors' information}

KA was born on September 1971 in Bonab, Iran. He gained his BSc degree in Electronics Engineering from the State University of Urumieh, Iran, in 1994 and his MSc from the University of Tarbiat Modarres, Iran, in 1997. Since 1998, he is a Faculty member (instructor) of the University of Tabriz, Iran. Then, he received his PhD in optical integrated devices in 2007. Now, he is an assistant professor in the University of Tabriz. MHA was born in 1973 in Iran. He received his BSc degree in Electronics Engineering from the State University of Urumieh in Iran in 1997 and his MSc from the Islamic Azad University of Iran (Tabriz Branch) in 2009. Since 1998, he is employed in Tabriz Oil Refining Company as an engineer.

\section{Acknowledgments}

The authors wish to acknowledge Prof. Dr. Ali Rostami for his valuable consultations and detailed and helpful comments on the research. We also appreciate the effort of the editor for the detailed corrections and editions.

\section{Author details}

${ }^{1}$ School of Engineering Emerging-Technologies, University of Tabriz, Tabriz 51666, Iran. ²Tabriz Oil Refining Company, Tabriz-Azarshahr Freeway, Sardorud Forked Road, Tabriz, Iran.

Received: 6 July 2011 Accepted: 8 October 2012

Published: 14 May 2013

\section{References}

1. Wang, $\mathrm{C}, \mathrm{Chu}, \mathrm{X}$, Mingmei, W: Detection of $\mathrm{H}_{2} \mathrm{~S}$ down to ppb levels at room temperature using sensors based on $\mathrm{ZnO}$ nanorods. Sensors and Actuators B 113, 320-323 (2006)

2. Telle, HH, Angel Gonzalez, U, Donovan, RJ: Laser Chemistry Spectroscopy, Dynamics and Applications. Wiley, New York (2007)

3. Tamaki, J, Maekawa, T, Miura, N, Yamazoe, N: Cuo- $\mathrm{SnO}_{2}$ element for highly sensitive and selective detection of $\mathrm{H}_{2} \mathrm{~S}$. Sens. Actuators B 9, 197-203 (1992)

4. Vasiliev, RB, Rumyantseva, MN, Yakovlev, MN, Gaskov, AM: $\mathrm{CuO} / \mathrm{SnO}_{2}$ thin film heterostructures as chemical sensors to $\mathrm{H}_{2} \mathrm{~S}$. Sens. Actuators B 50, 186-193 (1998) 
5. Chowdhuri, A, Sharma, P, Gupta, V, Sreenivas, K, Rao, KV: $\mathrm{H}_{2} \mathrm{~S}$ gas sensing mechanism of $\mathrm{SnO}_{2}$ films with ultra-thin $\mathrm{CuO}$ dotted islands. J. Appl. Phys. 92(4), 2172-2180 (2002)

6. Chowdhuri, A, Gupta, V, Sreenivas, K, Kumar, R, Mozumdar, S, Patanjali, PK: Response speed of $\mathrm{SnO}_{2}$ based $\mathrm{H}_{2} \mathrm{~S}$ gas sensors with $\mathrm{CuO}$ nanoparticles. Appl. Phys. Lett. 84(7), 1180-1182 (2004)

7. Kumar, R, Khanna, A, Tripathi, P, Nandedkar, RV, Potdar, SR, Chaudhari, SM, Bhatti, SS: $\mathrm{CuO}-\mathrm{SnO}_{2}$ element as hydrogen sulfide gas sensor prepared by a sequential electron beam evaporation technique. J. Appl. Phys. 36, 2377-2381 (2003)

8. Sarala Devi, G, Manorama, S, Rao, VJ: Gas sensitivity of $\mathrm{SnO}_{2} / \mathrm{CuO}$ heterocontacts. J. Electrochem. Soc. 142, 2754-2756 (1995)

9. Wagh, MS, Patil, LA, Seth, T, Amalnerkar, DP: Surface cupricated $\mathrm{SnO}_{2}-\mathrm{ZnO}$ thick films as a $\mathrm{H}_{2} \mathrm{~S}$ gas sensor. Mater. Chem. Phys. 84, 228-233 (2004)

10. Yamazoe, N, Matsushima, S, Maekawa, T, Tamaki, J, Miura, N: Control of Pddispersion in $\mathrm{SnO}_{2}$ based sensors. Meas. Sci. Technol. 1, $201-205$ (1991)

11. Jain, GH, Patil, LA, Wagh, MS, Patil, DR, Patil, SA, Amalnerkar, DP: Surface modified $\mathrm{BaTiO}_{3}$ thick film resistors as $\mathrm{H}_{2} \mathrm{~S}$ gas sensor. Sens. Actuators $B$ 117, 159-165 (2006)

12. Lantto, $\mathrm{V}$, Romppainen, $\mathrm{P}$ : Response of some $\mathrm{SnO}_{2}$ gas sensors to $\mathrm{H}_{2} \mathrm{~S}$ after quick cooling. J. Electrochem. Soc. 135(10), 2550-2556 (1988)

13. Yuanda, W, Maosong, T, Xiuli, H, Yushu, Z, Guorui, D: Thin film sensors of $\mathrm{SnO}_{2}-\mathrm{CuO}-\mathrm{SnO}_{2}$ sandwich structure to $\mathrm{H}_{2} \mathrm{~S}$. Sens. Actuators $\mathrm{B}$ 79, 187-191 (2001)

14. Tamaki, J, Yamada, Y, Yamamoto, Y, Matsuoka, M, Ota, I: Sensing properties of dilute hydrogen sulfide of $\mathrm{ZnSb}_{2} \mathrm{O}_{6}$ thick film prepared by dip coating method. Sens. Actuators B 66, 70-73 (2000)

15. Dong, C, Wenqing, L, Yujun, Z, Jianguo, L, Ruifeng, K, Min, W, Jiuying, C, Yiben, $\mathrm{C}: \mathrm{H}_{2} \mathrm{~S}$ detection by tunable diode laser absorption spectroscopy. Paper presented in the IEEE International Conference on Information Acquisition, Shandong (2006). 20-23 August

16. Robinson, JW, Skelly Frame, EM, Frame II, GM: Undergraduate Instrumental Analysis, 6th edn. Marcel Dekker, New York (2005)

17. Kauppinen, J, Partanen, J: Fourier Transforms in Spectroscopy, 1st edn. Wiley-VCH Verlag GmbH, Germany (2001)

18. Abbasian, K, Rostami, A, Koozeh Kanani, ZD: All-optical tunable devices and systems: a proposal for all-optical tunable mirror using electromagnetically induced transparency (steady state analysis). PIER 5, 25-41 (2008)

19. Yadipour, R, Abbasian, K, Rostami, A, Koozeh Kanani, ZD: A novel proposal for ultra-high resolution and compact optical displacement sensor based on electromagnetically induced transparency in ring resonator. PIER 77, 149-170 (2007)

doi:10.1186/2228-5326-3-34

Cite this article as: Abbasian and Abdollahi: Electromagnetically induced transparency-based gas detector design using Michelson

interferometer. International Nano Letters 2013 3:34.

\section{Submit your manuscript to a SpringerOpen ${ }^{\odot}$ journal and benefit from:}

- Convenient online submission

- Rigorous peer review

- Immediate publication on acceptance

- Open access: articles freely available online

- High visibility within the field

- Retaining the copyright to your article

Submit your next manuscript at $\gg$ springeropen.com 